\title{
The biopsychosocial model of stress in adolescence: self-awareness of performance versus stress reactivity
}

\section{Citation}

Rith-Najarian, Leslie R., Katie A. McLaughlin, Margaret A. Sheridan, and Matthew K. Nock. 2014. "The Biopsychosocial Model of Stress in Adolescence: Self-Awareness of Performance Versus Stress Reactivity." Stress 17 (2) (February 19): 193-203. doi:10.3109/10253890.2014.891102.

\section{Published Version}

doi:10.3109/10253890.2014.891102

\section{Permanent link}

http://nrs.harvard.edu/urn-3:HUL.InstRepos:33461083

\section{Terms of Use}

This article was downloaded from Harvard University's DASH repository, and is made available under the terms and conditions applicable to Open Access Policy Articles, as set forth at http:// nrs.harvard.edu/urn-3:HUL.InstRepos:dash.current.terms-of-use\#OAP

\section{Share Your Story}

The Harvard community has made this article openly available.

Please share how this access benefits you. Submit a story.

\section{Accessibility}




\title{
The biopsychosocial model of stress in adolescence: self- awareness of performance versus stress reactivity
}

\author{
Leslie R. Rith-Najarian ${ }^{1}$, Katie A. McLaughlin ${ }^{2,3}$, Margaret A. Sheridan ${ }^{3,4,5}$, and Matthew K. \\ Nock $^{5,6}$ \\ ${ }^{1}$ Department of Psychology, University of California - Los Angeles, Los Angeles, CA, USA \\ ${ }^{2}$ Department of Psychology, University of Washington, Seattle, WA, USA \\ ${ }^{3}$ Division of Developmental Medicine, Boston Children's Hospital, Boston, MA, USA \\ ${ }^{4}$ Department of Pediatrics, Harvard Medical School, Boston, MA, USA \\ ${ }^{5}$ Harvard Center for the Developing Child, Cambridge, MA, USA \\ ${ }^{6}$ Department of Psychology, Harvard University, Cambridge, MA, USA
}

\begin{abstract}
Extensive research among adults supports the biopsychosocial (BPS) model of challenge and threat, which describes relationships among stress appraisals, physiological stress reactivity, and performance; however, no previous studies have examined these relationships in adolescents. Perceptions of stressors as well as physiological reactivity to stress increase during adolescence, highlighting the importance of understanding the relationships among stress appraisals, physiological reactivity, and performance during this developmental period. In this study, 79 adolescent participants reported on stress appraisals before and after a Trier Social Stress Test in which they performed a speech task. Physiological stress reactivity was defined by changes in cardiac output and total peripheral resistance from a baseline rest period to the speech task, and performance on the speech was coded using an objective rating system. We observed in adolescents only two relationships found in past adult research on the BPS model variables: (1) pre-task stress appraisal predicted post-task stress appraisal and (2) performance predicted posttask stress appraisal. Physiological reactivity during the speech was unrelated to pre- and post-task stress appraisals and to performance. We conclude that the lack of association between post-task stress appraisal and physiological stress reactivity suggests that adolescents might have low selfawareness of physiological emotional arousal. Our findings further suggest that adolescent stress appraisals are based largely on their performance during stressful situations. Developmental implications of this potential lack of awareness of one's physiological and emotional state during adolescence are discussed.
\end{abstract}

\section{(c) 2014 Informa UK Ltd.}

Correspondence: Leslie R. Rith-Najarian, Department of Psychology, University of California - Los Angeles, 1193 Franz Hall, Los Angeles, CA 90095, USA. Tel: 1218766 5914. leslierrn@ucla.edu.

Declaration of interest

The authors report no conflicts of interest. The authors alone are responsible for the content and writing of the article. 


\section{Keywords}

Appraisal; cardiovascular; challenge; physiology; speech; Trier social stress test; threat

\section{Introduction}

Adolescence is a developmental period accompanied by significant environmental, physiological, cognitive, and neurobiological changes (Spear, 2009; Steinberg, 2005; Steinberg \& Morris, 2001). Environmental changes that disrupt homeostasis are typically referred to as stressors (Monroe, 2008). Many new stressors are introduced in adolescence, such as school achievement demands, family dynamic shifts, and romantic relationships (Grour et al., 1992; Seiffge-Krenke et al., 2001; Steinberg \& Morris, 2001). Performancerelated stressors such as standardized tests, class grades, and extracurricular activities emerge as meaningful stressors in adolescence (Denscombe, 2000; Phelan et al., 1994). Relative to children at earlier developmental periods, adolescence is not only a time during which more negative events are encountered (Larson \& Ham, 1993), but also a time of increased perceptions of stress in response to these stressors (Larson \& Ham, 1993; Spear, 2009). Moreover, adolescents experience increased reactivity in both the autonomic nervous system (ANS) and hypothalamic-pituitary-adrenal (HPA) axis in response to social and performance stressors as compared to younger children (Gunnar et al., 2009; Stroud et al., 2009). Thus, adolescents not only perceive more stressors than in previous developmental periods, but are also more reactive to those stressors, both emotionally and physiologically (Larson \& Ham, 1993).

What is then primarily responsible for the increased reporting of stress during adolescence? First, the increase could simply be due to the fact that there are more social and environmental demands stressors during adolescence. Alternatively, adolescents might be more likely to perceive or appraise situations as more stressful than at earlier developmental periods. The stress response refers to an individual's affective, cognitive, behavioral, and biological responses involved in regaining psychological and physiological balance after disrupted homeostasis (Schneiderman et al., 2005). Thus, greater perceptions of stress might result from any one of these components of the stress response. Biologically, increased perceptions of stress could be related to the increases in physiological and emotional reactivity to stressors in adolescence (Gunnar et al., 2009; Larson \& Ham, 1993; Stroud et al., 2009). Cognitively, it could be due to increasing ability to contemplate abstract and distal rewards and consequences of social and environmental changes (Davey et al., 2008), as metacognitive abilities and cognitive control abilities are still developing in adolescence (Kopp, 1989; Ordaz \& Luna, 2012; Sowell et al., 2002; Steinberg, 2005). Finally, adolescents might behave differently in social or evaluative situations in ways that lead to greater perceptions of stress.

In adults, cognitive, biological, and behavioral responses have been found to relate to one another in reliable ways (Blascovich et al., 1999, 2001; Folkman et al., 1986), however, the relationships between these response systems during adolescence have rarely been studied. Here, we examine the relationships between cognitive, physiological, and behavioral 
responses to an evaluative, performance situation in adolescents and compare these relationships to what is known to occur in adulthood.

The biopsychosocial (BPS) model of challenge and threat is the prevailing theoretical framework linking cognitive (i.e. stress appraisals), physiological (i.e. ANS reactivity), and behavioral (i.e. performance) responses to performance stress in adults (Blascovich et al., 2001). The BPS model posits that during an acute stressor, individuals first make a pre-task stress appraisal of the situation that involves mentally weighing one's personal resources (i.e. skills, intelligence, knowledge) against the demands of the situation (i.e. what will be required to succeed) (Blascovich et al., 1999; Folkman et al., 1986). This initial appraisal shapes the physiological response to the stressful situation. Within the BPS model, physiological reactivity is categorized by the efficiency of the ANS response to the stressor (Blascovich \& Tomaka, 1996; Mendes et al., 2003). Physiological responses then influence situational performance, referring to how effectively one deals with the task (Blascovich et al., 1999; Mendes et al., 2003, 2008). Once the situation resolves, these factors together shape a post-task stress appraisal that incorporates perceptions of the degree to which the situation was stressful (Quigley et al., 2002).

By sequentially relating these variables the BPS model distinguishes between two types of stress responses in adults: challenge versus threat responses (Blascovich \& Tomaka, 1996; Mendes et al., 2003). A challenge response begins with an appraisal that one has the necessary personal resources to cope with the demands of the situation. This elicits a physiological challenge response - a pattern of efficient ANS reactivity - which in turn facilitates performance, culminating in a post-task stress appraisal that the situation was a positive challenge rather than a stressor (Blascovich et al., 1999; Mendes et al., 2003, 2008). Conversely, a threat response begins with an appraisal that the demands of the situation exceed one's resources to cope, which elicits a physiological threat response - a pattern of inefficient ANS reactivity - that inhibits performance and leads to a post-task appraisal that the situation was stressful and threatening. Figure 1 outlines the relationships between stress appraisals, physiological reactivity, and performance in the BPS model.

The relationships described in the BPS model have been supported in a variety of studies examining performance situations in adults. Several studies found that undergraduate students who appraised a mental arithmetic task to be less threatening had a higher percentage of correct responses and were more likely to exhibit a physiological challenge response than those who perceived the task to be threatening (Kelsey et al., 2000; Schneider, 2008; Tomaka et al., 1993). Participants who performed better on the mental arithmetic task also reported lower stress appraisal following the task (Kelsey et al., 2000; Tomaka et al., 1993). Tomaka et al. (1993) also found evidence for self-awareness of performance ability; participants who performed poorly also self-rated their performance as low and reported more post-task perceptions of stress. Support for the BPS model has also been found in other performance situations, including athletics, standardized testing, and complex prediction tasks (Blascovich et al., 2004; Jamieson et al., 2010; Drach-Zahavy \& Erez, 2002). It is believed that performance facilitation and inhibition effects, described above, occur as a result of interpreting the emotions that are elicited from a challenge or threat state (Jones et al., 2009; Moore et al., 2012). Individuals who experience a physiological threat response 
are more likely to associate it with emotional anxiety and to interpret that anxiety as debilitating, as compared to individuals who experience a physiological challenge response (Quested et al., 2011; Williams et al., 2010). Evidence for consistent associations of stress appraisals with physiological stress reactivity and performance indicates that, in adults, cognitive, physiological, and behavioral responses to stress are consistent and aligned with one another.

Surprisingly, the BPS model has rarely been examined outside of adulthood. We address this gap in the existing literature by examining the BPS model in adolescents. We examined whether the associations between stress appraisals, physiological reactivity, and performance in adolescents resemble the well-established patterns observed in adults. To do so, we administered the Trier Social Stress Test (TSST), a procedure that has highperformance demands and reliably elicits both ANS and HPA axis responses in adolescents (Gunnar et al., 2009; Kudielka et al., 2007; Stroud et al., 2009). The TSST has been widely used in previous studies of the BPS model in adults (e.g. Jamieson et al., 2012).

The first goal of this study was to examine the relationships between stress appraisals (preand post-task) and physiological reactivity in adolescents. Because adolescents have not fully developed the cognitive capacities that underlie accurate emotional awareness (Ordaz \& Luna, 2012; Sowell et al., 2002; Steinberg, 2005), we hypothesized that associations between stress appraisals and physiological reactivity would be relatively weak in adolescents as compared to what has been observed in adult studies.

The second goal was to investigate whether performance during the TSST, as rated independently by an outside observer, was influenced by pre-task stress appraisal and physiological reactivity. As reviewed above, the relationship between physiological reactivity and performance is contingent upon self-awareness of one's emotional and physiological state. Because we expect that these awareness processes are less mature in adolescents, we hypothesize that the relationship of performance to pre-task stress appraisal and physiological reactivity would be weaker in adolescents than what has typically been observed in adult studies.

The third goal of this study was to identify predictors of post-task stress appraisal, ultimately to identify what aspects of stress responses contribute to subjective experiences of stress in adolescents. As described above, adolescents might be relatively unaware of their physiological state, and thus, rely on other cues to interpret their emotional response to a stressor. Considering the variables in the BPS model, performance could be one such factor. Indeed, adolescents generally have accurate perceptions of how well they performed on a task, such as with an athletic or musical task (Allen \& Howe, 1998; Hewitt, 2005). This suggests that perception of performance ability might be more concrete and tangible for adolescents than interpreting their degree of physiological arousal or emotional distress. We thus hypothesized that task performance would be positively associated with post-task stress appraisal and would be more strongly related with post-task appraisals than physiological reactivity. 


\section{Method}

\section{Participants}

Our sample was comprised of 87 adolescents who participated in the context of a larger study approved by the Institutional Review Board of Boston Children's Hospital. Of these, seven participants were excluded for unusable physiological data due to equipment problems and one participant was excluded for having incomplete questionnaires. The final analytic sample included 79 participants (49 female, 30 male) between the ages of 13 and 17 ( $M$ $=14.73, S D=1.30$ ). Participants were recruited with flyers at hospitals, school programs, and public spaces in Cambridge and Boston, MA. Recruitment was aimed at producing a sample with high racial/ethnic and socioeconomic variation. Participant self-reported ethnicities were: White $(n=34 ; 43.0 \%)$, Biracial $(n=18 ; 22.8 \%)$, African America $(n=12 ; 15.2 \%)$, Hispanic $(n=7 ; 8.9 \%)$, Asian $(n=6 ; 7.6 \%)$, and other $(n=2 ; 2.5 \%)$.

\section{Procedures}

Stress induction-The TSST was used to create a stressful performance situation (Buske-Kirschbaum et al., 1997; Kirschbaum et al., 1993). The TSST includes an anticipation period and a test period in which participants are asked to deliver a speech for an evaluating audience. In this study, adolescents were asked to give a 5-min speech about the qualities of being a good friend and which of those qualities they personally do and do not have. Participants were told that they would be evaluated by experts in verbal and nonverbal communication and that it was important to perform well. After a 5-min preparation period, adolescents delivered the speech in front of two "evaluators," one male and one female. Evaluators were confederates trained to provide only neutral or negative non-verbal feedback (i.e. negative facial expressions, appearing bored, taking notes, absence of affirmative nodding). The TSST also includes a surprise math task that occurs following the speech. However, participants did not complete pre-task appraisal items about the math task because they were unaware that there would be a math task. Because the math task has no pre-task stress appraisal measure - an important component of the BPS model - our analysis focuses on the speech component only. The TSST has shown to effectively elicit physiological responses in the ANS and HPA-axis in adolescents (Gunnar et al., 2009; Kudielka et al., 2007; Stroud et al., 2009).

Study protocol-Participants arrived with their parents at the laboratory and written consent and assent were obtained from parents and adolescents, respectively. Participants and their parents completed pre-session questionnaires in separate rooms. After attaching the necessary sensors, ANS activity was recorded during an initial baseline resting period. Next, the experimenter began the TSST by explaining the speech task and informing the participant that they would be evaluated by two professionals and that their performance would be videotaped and viewed by other experts later. After meeting the two "evaluators," participants completed a pre-task questionnaire assessing their emotional state and appraisals about the upcoming task. Following the speech preparation period, the evaluators re-entered the room for the participant's speech delivery. Audio and video recordings were taken of participant's speech during the TSST, and ANS activity was recorded. Following the speech, participants completed a post-task questionnaire. Before leaving, participants 
were extensively debriefed to ensure they understood that the evaluators' responses were unrelated with their performance and they were introduced to the evaluators, who provided positive feedback about the participant's performance.

Physiological data collection-Continuous cardiac and hemodynamic measures were recorded noninvasively according to accepted guidelines (Sherwood et al., 1990). Electrocardiogram (ECG) recordings were obtained with a Biopac ECG amplifier (Goleta, CA) using a modified Lead II configuration (right clavicle, left lower torso, and right leg ground). Cardiac impedance recordings were obtained with a Bio-Impedance Technology model HIC-2500 impedance cardiograph (Chapel Hill, NC). One pair of Mylar electrode tapes were placed on the neck and another pair were placed on the torso. A continuous 500 $\mu \mathrm{A}$ AC $95 \mathrm{kHz}$ current was passed through the two outer electrodes, and basal thoracic impedance $(\mathrm{zO})$ and the first derivative of basal impedance $(\mathrm{dz} / \mathrm{dt})$ was measured from the inner electrodes. Basal impedance provides a measure of blood flow in the thoracic cavity. Acknoweldge software and Biopac MP150 hardware and was used to integrate and acquire the ECG and impedance cardiography data, both of which were sampled at $1.0 \mathrm{kHz}$. A Colin Prodigy II oscillometric blood pressure machine (Colin Medical Instruments, San Antonio, TX) was used to record blood pressure. ECG and impedance cardiograph were scored by trained personnel following acquisition using Mindware Software (Mindware Technologies, Gahanna, $\mathrm{OH}$ ) in order to calculate heart rate (HR) and stroke volume (SV) values. ${ }^{1}$ Cardiac output $(\mathrm{CO})$ for each minute was calculated using the standard formula $(\mathrm{SV} \times \mathrm{HR}) / 1000$. We also calculated total peripheral resistance (TPR) using the standard formula (Mean Arterial Pressure/CO) $\times 80($ Sherwood et al., 1990) .

Performance video coding - A performance measure - the Evaluated Speech Performance Measure (ESPM) - was created to evaluate performance during the speech component of the TSST based on previous studies using the TSST and similar social speech tasks (Fydrich et al., 1998; Gray et al., 2008; Hodgins et al., 2010; Willard \& Gramzow, 2009). One male and one female performance coder separately watched the video recordings and scored participants on a variety of dimensions (described in more detail in the Measures section). The first author (L.R.R.N.) served as the female performance coder for all participants, and four male performance coders were each assigned one-fourth of the participants. All coders were undergraduate research assistants trained by the first author. After the video coding manual was verbally reviewed, coders were trained to reliability on the ESPM using practice videos from pilot subjects. Coders used practice videos until they produced four consecutive scores that were reliable with the first author's coding. The coders completed their remaining subset of participants independently and were blind to the ratings of the first author during coding.

\footnotetext{
${ }^{1}$ All signals were averaged into one-minute epochs and the ensembled data were then visually inspected and scored. HR data scoring involved proper identification of the R-point and removal of artifacts to allow quantification of HR. Impedance cardiography data scoring involved proper identification of the B-point (opening of the aortic valve), the Z-point (peak of the dz/dt waveform) and the Xpoint (closing of the aortic valve) on the dz/dt waveform. Identification of these points allows quantification of stroke volume (SV), the amount of blood ejected from the heart on each cardiac cycle. Because accurate scoring of impedance cardiography data requires manual placement of the B point (Blascovich et al., 2011), these data were scored by two independent raters. SV differences of more than $5 \%$ were reviewed and adjudicated by the second author (K.A.M.).
} 
Physiological reactivity-ANS measures of stress reactivity differentiating between physiological challenge and threat responses in the BPS model include CO and TPR. The challenge ANS activity profile is characterized by increased sympathetic nervous system activity (elevated HR, shorter pre-ejection period) with decreased vascular resistance, resulting in increased $\mathrm{CO}$ and representing a state of cardiac efficiency (Blascovich et al., 1999). The threat ANS activity profile is characterized by increased sympathetic nervous system activity (elevated HR, shorter pre-ejection period) with increased vascular resistance, resulting in relatively less increase in $\mathrm{CO}$ and representing a state of cardiac inefficiency (Blascovich et al., 1999). The differentiation of a challenge versus threat cardiovascular response is based on changes (from baseline to task) in CO and TPR (Blascovich et al., 1999; Mendes et al., 2003, 2008). Accordingly, we calculated change scores for CO and TPR from the first minute of the baseline period to the first minute of the speech task, when physiological activation is highest (Jamieson et al., 2012). When physiological data from these minutes was missing or implausible, data from the closest minute were substituted. For three participants we substituted blood pressure values from minute 4 of the speech task for minute 1 of the speech task due to problems with the blood pressure cuff. Because there is no established "cut-off" for determining how much of an increase or decrease in CO and TPR indicates a challenge or threat response, we used continuous measures of reactivity, consistent with prior research (Mendes et al., 2001). Higher $\triangle \mathrm{CO}$ and lower $\triangle \mathrm{TPR}$ scores represent more challenge stress reactivity, whereas lower $\Delta \mathrm{CO}$ and higher $\Delta \mathrm{TPR}$ scores represent more threat stress reactivity (Blascovich et al., 1999).

Stress appraisals-Participants were asked to provide appraisals of the stressfulness of the task before and after the speech. Using these ratings, we examined pre-task stress appraisal (anticipated stress) and post-task stress appraisal (subjective stress recalled). Stress appraisals were operationalized by a total score calculated from 3 items on the task questionnaire. Participants completed appraisals of the degree of stress ("The upcoming task is very stressful"), threat ("I think the upcoming task represents a threat to me"), and positive challenge ("I view the upcoming task as a positive challenge") that they anticipated to experience during the speech using a measure utilized in previous studies of the BPS model (Jamieson et al., 2012; Mendes et al., 2007). Participants completed the same questions after completing the speech ("The speech was very stressful"; "I saw the speech as representing a threat to me"; "I saw the speech as a positive challenge"). Each item was rated on a 1-7-point scale. These 3 items were added together, with the positive challenge item reverse scored. Higher scores on the questionnaire items represented a greater stress appraisal of threat with a score of 3 being the lowest possible and 21 being the highest possible.

Performance-Performance quality was operationalized by averaging the scores of the two raters on the ESPM. The ESPM included 11 items assessing speech energy, speech continuity, body presentation, and verbal communication. Speech energy was assessed with 4 items coding the number of explaining gestures to enhance the speech, the volume of the participant's voice, and how many positive and negative facial expressions the participant 
displayed. Each item in this subcategory was scored on a 5-point scale. Speech continuity was scored by counting the number of pauses of five or more seconds and noting if the participant did not continue the speech for the full $5 \mathrm{~min}$. This item was reverse scored such that participants earned more points for pausing less frequently. The subcategory of body presentation included 3 items that assessed how much the participant displayed poise versus discomposure. Items included ratings of the degree of tension in the body, the degree of closed posture, and fidgety behavior and were each scored on a 5-point scale. Verbal communication included 3 items that assessed how much the participant's speech adhered to the instructions they were given about how to give a good speech. These items assessed speech organization, confidence of presentation, and quality of speech content. Each item in this category was scored on a 7-point scale. Higher total ESPM scores represented a better speech performance by participant, with a score of 11 being the lowest possible and 61 being the highest possible score.

The ESPM items demonstrated good reliability in this sample $(a=0.84)$. Inter-rater reliability between the female and male coders was excellent ( $r=0.89, p<0.001$ for all male coders combined; $r=0.86-0.95$ across the four male-female pairs).

\section{Data analysis}

Linear regression was used to evaluate the associations between pre-task stress appraisal, stress reactivity, performance, and post-task stress appraisal. We first evaluated the association between pre-task and post-task stress appraisal. Two models were estimated to predict stress reactivity, one with $\triangle \mathrm{CO}$ as the dependent variable and one with $\triangle \mathrm{TPR}$ as the dependent variable. ${ }^{2}$ We next examined the associations of pre-task appraisal and stress reactivity with speech performance. Finally, we examined post-task stress appraisals using a hierarchical regression model. Step 1 included pre-task stress appraisal, Step 2 added stress reactivity (either $\Delta \mathrm{CO}$ or $\Delta \mathrm{TPR}$ ), and Step 3 added the speech performance score.

\section{Results \\ Descriptive statistics}

The descriptive statistics for pre-task appraisal, post-task appraisal, performance, baseline HR, CO, TPR, and speech HR, CO, and TPR are reported in Table 1.

\section{Manipulation check}

We examined changes in HR from the baseline rest period to the speech task to ensure that the TSST was experienced as a stressful situation and that participants were engaged in the

\footnotetext{
${ }^{2}$ Participants were also filtered for potential race-related physiological responses. Race-related physiological responses were defined as potential false positive challenge physiological responses, resulting from participant's feelings of frustration, due to perceived racial discrimination by the evaluators during the Trier Social Stress Test. Mendes et al. (2008) found that negative feedback from an evaluator of different race produced a anger cardiovascular response in the participant that imitates challenge-patterned reactivity. They concluded that an anger response due to perceived racism cannot be differentiated from a true challenge response, because both result in increased ventricular contractility and HR. Participants were identified based on three characteristics: (1) responses on the post-task questionnaire (high upset/hostile emotions, high agreement with evaluator attribution statements such as "He/she had a bias against me"); (2) differing race from the evaluators; and (3) challenge physiological response (high $\Delta \mathrm{CO}$, low $\Delta \mathrm{TPR}$ ). Five participants matched this profile and were coded into a data analysis filter. Statistical results from preliminary data analysis were consistent when these five participants were included and excluded, so the decision was made by the first author to include these five participants in the final data analysis.
} 
task (Blascovich et al., 2004). The mean change in HR ( $M=27.21 \mathrm{bpm}, S D=17.84)$ was significantly greater than zero, $t(79)=13.56, p<0.001$, indicating that participants responded physiologically to the TSST.

\section{Between-group differences}

First, analyses were conducted to test if sex, race/ethnicity, or age predicted differences in baseline levels of physiological variables between participants. An independent $t$-test of sex revealed no differences between mean baseline $\mathrm{CO}, t=0.59, p=0.56$. A one-way ANOVA of race revealed a marginal difference between racial/ethnic groups in mean baseline $\mathrm{CO}, F$ $=2.12, p=0.07$; however, a post-hoc Bonferroni test revealed that none of the racial/ethnic groups significantly differed from each other. A one-way ANOVA of age revealed a marginal difference in $\mathrm{CO}$ across age, $F=2.37 p=0.06$, with older participants exhibiting higher $\mathrm{CO}$; however, again a post-hoc Bonferroni test revealed that none of the age groups significantly differed from each other. Nevertheless, age, sex, and race/ethnicity were included as control variables in all subsequent analysis.

\section{Testing the biopsychosocial model}

Pre-task stress appraisal-Participants reported higher post-task stress appraisals ( $M$ $=12.16, S D=4.12)$ than pre-task appraisals $(M=10.57, S D=4.20)$. The mean change in stress appraisal $(M=1.59, S D=2.82)$ was significantly greater than zero, $t(79)=5.03$, $p<0.001$, indicating that the TSST was associated with significant increases in perceived stress.

The difference between pre- and post-task stress appraisal scores was also significant in a repeated measures ANOVA with a Greenhouse-Geisser correction, $F(1,78)=25.32$, $p<0.001$, and again in post-hoc tests using the Bonferroni correction, $p<0.001$. Pre-task stress appraisal was significantly associated with post-task stress appraisal, $B=0.78$, $p<0.001, R^{2}=0.61$. Pre-task stress appraisal was included as a control variable in all subsequent analysis predicting post-task stress appraisals.

Physiological reactivity-In a linear regression predicting change in $\mathrm{CO}(\triangle \mathrm{CO} ; M$ $=0.77, S D=1.15$ ) from baseline to the speech task based on pre-task stress appraisal, no association was observed between pre-task appraisal and changes in CO, $B=-0.001, p$ $=0.99, R^{2}=0.07$. Similarly, no association was observed between pre-task stress appraisal and changes in TPR ( $\triangle \mathrm{TPR} ; M=137.45, S D=293.04$ ) from baseline to the speech, $B=0.11$, $p=0.36, R^{2}=0.03$.

Speech performance-We next tested how our performance variable related to pre-task stress appraisal and physiological reactivity. In a linear regression predicting speech performance ( $M=36.37, S D=9.19)$, neither pre-task appraisal $(B=-0.12, p=0.33)$ nor stress reactivity variables ( $\Delta \mathrm{CO}, B=-0.02, p=0.87 ; \Delta \mathrm{TPR}, B=0.16, p=0.27$ ) were associated with performance ability, $R^{2}=0.11$.

Post-task stress appraisal-We conducted two hierarchical multiple regressions predicting post-task stress appraisal from pre-task appraisal, physiological reactivity, and 
speech performance. The first model examined changes in $\mathrm{CO}$ as the indicator of physiological reactivity, shown in Table 2 . In this model, changes in $\mathrm{CO}$ were unrelated to post-task stress appraisal controlling for pre-task appraisal, $B=0.09, p=0.23, R^{2}=0.62$.

Speech performance was added at the next step of the model and was negatively associated with post-task stress appraisal, $B=-0.19, p=0.01, R^{2}=0.65$, such that higher performance scores were associated with lower post-task appraisals, controlling for pre-task appraisal and changes in $\mathrm{CO}$. In the second hierarchical multiple regression predicting post-task appraisal, shown in Table 3, changes in TPR were unassociated with post-task appraisal controlling for pre-task appraisal, $B=0.02, p=0.85, R^{2}=0.61$. Speech performance was negatively associated with post-task stress appraisal, $B=-0.20, p=0.01, R^{2}=0.65$ controlling for pretask appraisal and changes in TPR.

In an additional supplementary correlation analysis performance scores were related to adolescents' responses on a separate post-task questionnaire item "I had the abilities to perform the speech successfully" $(r=0.56, p<0.001)$.

\section{Variation across age}

We conducted an additional linear regression including interaction terms with age and each of the variables of the BPS model. The results are shown in Tables 4 and 5. The analysis revealed that age was not a moderator of any reported associations as none of the interaction terms were significant in the CO linear regression, all $p>0.30$, or in the TPR linear regression, all $p>0.50$.

\section{Discussion}

Although cognitive, physiological, and behavioral responses to stress each contribute to perceptions of stress in adults, the relationships among these components of stress responses have rarely been examined in adolescents, a time period characterized by increased stress exposure and reactivity. To address this gap in knowledge, we used the BPS model to examine the relationships among cognitive, physiological, and behavioral stress responses in a sample of adolescents who completed the TSST. Our first goal was to evaluate whether physiological stress reactivity relates to stress appraisals (pre- and post-task) in adolescents, as these two facets of the stress response are aligned in adults (Kelsey et al., 2000; Schneider, 2008; Tomaka et al., 1993). Due to the fact that, relative to adults, adolescents are less able to hold in mind future events, we expected weak relationships between variables. Consistent with our hypothesis, we observed that physiological stress reactivity was neither predicted by pre-task stress appraisals, nor did it predict post-task stress appraisal. Our second research goal was to investigate whether performance was influenced by pre-task stress appraisal and/or stress reactivity. Again, consistent with our hypothesis, but in deviation from findings with adults, we observed that these variables were not related to performance in our adolescent population. Finally, the third goal of this study was to identify predictors of post-task stress appraisal. Consistent with our hypothesis, performance, instead of stress reactivity, was most strongly related to post-task stress appraisal. 
Our findings highlight unique developmental differences in the relationships between stress appraisals, physiological reactivity, and performance in adolescents. Specifically, they indicate that many of the relationships between cognitive, physiological, and behavioral responses to stressors in the BPS model that have been observed in adults (e.g. Kelsey et al., 2000; Tomaka et al., 1993) do not apply to adolescents, shown in Figure 2. The BPS model posits that pre-task appraisals are associated with physiological reactivity and performance, all three of which predict appraisals following the stressor. Only two of these relationships were supported in adolescents: (1) pre-task stress appraisal predicted post-task stress appraisal - which indicates that the TSST successfully created a stressful situation (Kassam et al., 2009); and (2) performance predicted post-task stress appraisal. This suggests not that the associations among variables in the BPS model are completely different in adolescents, but rather that the relationships with stress reactivity are weaker, suggesting developmental variation in the tenets of the BPS model.

One of the primary deviations in our findings from what the BPS model predicts is that physiological reactivity was unrelated to cognitive and behavioral aspects of stress responses. First, pre-task stress appraisal was unrelated to physiological reactivity. Specifically it was unrelated to changes in either CO or TPR, the two variables used to distinguish between challenge and threat physiological responses to stress in the BPS model (Blascovich \& Tomaka, 1996; Mendes et al., 2003, 2008). Furthermore, stress reactivity did not predict post-task stress appraisal. Together these finding suggest that adolescents might have difficulty predicting and appraising their physiological and emotional responses to stress. This does not necessarily mean that adults are always more capable, as there is individual variation in ability to accurately appraise their physiology (e.g. Sze et al., 2010), but relative to adults in general, adolescents appear to report stress appraisal in a manner that is less aligned with their physiological reactivity.

Why might physiological reactivity be unrelated to cognitive appraisals in adolescents? We believe that this lack of association could represent poor emotional self-awareness, possibly due to immaturity of neurobiological systems underlying aspects of self-awareness as well adolescent's relative inexperience with their heightened physiological reactions to stressors. The neural systems that regulate self-awareness of physiological responses are still developing in adolescents (Sowell et al., 2002), and poor self-reporting of physiological stress responses has been observed in adolescent populations (Stroud et al., 2009). The brain regions involved in decision-making and emotion processing are still maturing throughout adolescence (Gogtay et al., 2004), in particular areas of the medial prefrontal cortex and insular cortex that have been found to be involved in self-evaluation and self-awareness of one's affective states and physiological responses for adults (Ochsner et al., 2004; Ordaz \& Luna, 2012; Romeo \& McEwen, 2006; Sowell et al., 2002; Steinberg, 2005). The comparative lag in the development of neural regions underlying self-awareness and other complex cognitive functions could result in poor correspondence between cognitive appraisals and physiological responding in a time-limited fashion specifically during adolescence. In addition, the systems involved in stress responding, such as the ANS and HPA axis, are more reactive in adolescents than in children (Gunnar et al., 2009; McCormick \& Mathews, 2007; Stroud et al., 2009). However, adolescents may still be learning how to interpret and to respond to these heightened physiological responses. This 
may explain why research has found that compared to younger children, although adolescents exhibited increased physiological responses, they simultaneously did not report greater affective responses to these stressors (Stroud et al., 2009). The combination of relatively underdeveloped self-awareness processes and little self-knowledge could be driving adolescents' inability to interpret their stress arousal, and the result is that they can neither accurately predict their own perceptions of stress nor appraise their physiological reactions to stressors. These possibilities warrant examination in future studies in order to determine if self-awareness deficits are indeed involved in the associations between stress appraisals and physiological reactivity.

A second departure of our findings from the predictions of the BPS model involves performance. Neither pre-task stress appraisals nor physiological reactivity predicted performance during the speech, indicating that the performance inhibition/facilitation phenomenon seen in adults was not replicated with our adolescent sample. Specifically, research in adults has found performance to be related to both stress appraisal and physiological reactivity (e.g. Kelsey et al., 2000; Schneider, 2008; Tomaka et al., 1993), such that interpretation of a challenge or threat states precedes performance facilitation/ inhibition effects (Quested et al., 2011; Williams et al., 2010). This necessary self-awareness of one's emotional and physiological state could be one reason why adolescents did not experience the performance facilitation/inhibition effect that adults do. Our findings suggest that performance in adolescents might be relatively independent of stress appraisal and physiological reactivity, highlighting the importance of determining which factors are more strongly related to performance ability during this developmental period.

Although pre-task stress appraisal did not predict performance, performance was associated with post-task stress appraisal, such that adolescents who had higher scores on our objective measure of performance reported that the TSST was less stressful after the task was completed. This suggests that adolescents' performance on the task most directly informed their perception of the situation as stressful, accounting for the increase in post-task stress appraisal from pre-task, more so than their physiological response to the task. This pattern could suggest greater self-awareness of performance than of physiological reactivity in adolescence, and this is supported by the strong relationship we found between adolescents' objective performance scores and their post-task report of having the abilities to perform the speech successfully.

Thus, adolescents may rely on unique cues to interpret their subjective experiences of stress. Because adolescents may not have developed the skills to accurately perceive or interpret physiological responses to stress, they might look to other cues to interpret their emotions following a stressor. Post-task subjective stress experiences in all populations are believed to partially be a result of perceptions of poor performance (Sanders, 1983). For adolescents it might be more accessible to evaluate their performance than to assess their physiological response to a stressor. Previous research has demonstrated that adolescents can accurately evaluate performance in athletic and musical performance situations (Allen \& Howe, 1998; Hewitt, 2005), and that the concept of ability plays an important developmental role as adolescents, compared to younger children, perceive performance ability as an indicator of capacity relative to that of others (Nicholls, 1984). Specifically, the central manipulation in 
the TSST involves neutral and negative social feedback provided by evaluators, and evidence from numerous studies indicates that individuals are highly sensitive to this type of negative social feedback (Dickerson \& Kemeny, 2004). This negative social feedback draws attention to task performance, which is the basis for the social evaluation. When providing reports of internal states, our attention is captured more strongly by external stimuli than internal sensations particularly when there are more salient external cues (Pennebaker, 1982). The external markers of performance might be somewhat more objective and salient than markers of stress reactivity. For example, adolescents might notice the number of times they stopped speaking, the tone of their voice, the degree to which their voice trembled, or the quality of their argument. Therefore because adolescents exhibit difficulty recognizing internal states (Stroud et al., 2009), these types of external cues could be more accessible. Consistent with this, our results may suggest that adolescents are more able to accurately appraise how well they performed than they are able to appraise their physiological response, and their perceptions of performance most directly influence their self-reporting of stressful experiences.

\section{Limitations and future directions}

Several limitations of this study are important to acknowledge. First, it is unclear how the patterns observed here apply to other types of stressful situations. These relationships should be evaluated in other kinds of stressful tasks, such as time-pressured tasks or social interactions with peers (e.g. Stroud et al., 2009). In addition, the association between performance and stress appraisal and physiological reactivity might vary across performance on a variety of different tasks, such a math performance, athletic performance, tasks involving working memory, and so on (Schmader et al., 2008). Second, a wide range of other factors not measured in the study could have influenced the stress response variables we examined, such as individual differences in personality, cognition, and emotional response, as well as perceived social support of the evaluators (Seery, 2011; Wirtz et al., 2006). Third, although our measure of performance ability was reliable across raters in this study, the validity of the measure warrants examination in other samples. Fourth, the stress reactivity variable was created from the distribution of reactivity scores in our sample, as there is no objective standard cut-off point for defining challenge versus threat physiological stress reactivity scores; however, this is the standard used in previous studies of the BPS model (Blascovich et al., 1999; Mendes et al., 2001, 2008). We do not know if our findings are limited to the sympathetic branch of the ANS or if they could be generalized to other aspects of the physiological stress response (e.g. HPA axis), which is an important direction for future studies. Fifth, stress appraisals were measured by self-report, which can introduce problematic response biases. Reports of challenge and threat processes vary in accuracy, depending on how consciously the appraisal process occurs (Blascovich \& Mendes, 2000). For this study, we cannot be certain if adolescents are less conscious of their appraisals, or simply less able to articulate them. It is possible that adolescents comprehend "threat," "challenge," and "stress" differently, leading them to report stress appraisal differently than adults have in other studies. In addition, pre-task stress appraisals predicting post-task stress appraisals might also simply reflect similar responding patterns on each questionnaire (Howard \& Dailey, 1979). 
Finally, we examined only adolescents in this study, comparing their performance, stress appraisals, and physiological responses to the wealth of data available on adults. As we did not make a direct developmental comparison here, it could be that some other confounding factors account for the difference in findings between our studies and others in adulthood. We think this is unlikely because we use methods and equipment identical to those used in previous studies of adults; in addition, although we did not observe some previously reported findings, we did replicate others (e.g. change in stress appraisal before and after performance, the association between task performance and stress appraisal). We believe that our findings could possibly point to a differential relationship between physiological stress response and stress appraisal between adults and adolescents. Our finding that age was not a moderator was not surprising, as our sample included adolescents in a narrow age range spanning just four years (ages 13-17; all females were post-menarchal). Given this limited range, we would not expect to see substantial developmental variation across this period, but could still expect to see differences when comparing adults to adolescents. Our interpretations of our findings could benefit from future studies that are able to conduct such a direct comparison within the same study.

Our findings highlight the importance of examining variation in the relationships within the BPS stress model across development. It is unknown whether the relationships observed here would also be found in younger children or whether adolescence is a unique developmental period with regard to stress responses. Previous research on stress responses has been criticized for relying solely on college students (e.g. Schneider, 2008; Seery, 2011). Therefore studying the BPS model in new populations, as we have done here, will be important for future research.

Our finding that adolescents might be relatively unaware of their own physiological reactivity during stress could have implications for intervention programs addressing stressreduction during the teenage years. The development of emotional self-awareness is critical to one's ability to adaptively cope with the demands of stressors (Salovey et al., 1995; Zuddas, 2012), whereas an inability to identify one's emotions is associated with worse coping and more distress (Kerr et al., 2004). Fortunately, research has found that being able to re-appraise one's situation as less threatening promotes a more challenge physiological response (Jamieson et al., 2012). Because emotional awareness is an important component of interventions (Philippot \& Segal, 2009), program designs might benefit from including components to teach adolescents to more accurately appraise their responses to stress. Future intervention research could further elucidate the potential emotional awareness difficulties adolescents might have, and assess whether awareness can be increased and lead to adaptive re-appraisal.

Importantly, it remains unknown how this stress responding pattern in adolescence changes as adolescents transition into adulthood. Evidence suggests developmental canalization in stress responses such that individuals become more oriented towards either a challenge or threat pattern of stress response, both subjectively and physiologically (Moos \& Holahan, 2003; Seery, 2011). Because adolescents are still developing, it is important to investigate how their current stress responding predicts patterns of stress responding in adulthood. Adolescents do not appear to exhibit two separate patterns - challenge versus threat - as has 
been observed in adult studies on the BPS model (e.g. Blascovich et al., 2004), so they might instead more appropriately be categorized into four groups: challenge-reactivity/goodperforming (CG), threat-reactivity/good-performing (TG), challenge-reactivity/poorperforming (CP), threat-reactivity/poor-performing (TP). The CG and TP groups have comparatively more congruent stress appraisals. Considering that most adults have relatively more aligned cognitive, physiological, and behavioral stress responses, we consider three possibilities that might explain how the TG and CP groups develop. First, with time adolescents' physiological stress reactivity patterns might slowly become aligned with performance. Alternatively, adolescents' performance ability might slowly begin to match physiological reactivity, if physiological responses are persistent. If the former is true, this could be beneficial for the TG group and detrimental for the CP group. Conversely, if the latter is true, then this could be beneficial for the CP group and detrimental for the TG group. Currently research from adults seems to support the former possibility. For example, it was found that adults who had more positive challenge appraisals after a math tasks also made more positive challenge pre-task appraisals on a following math task (Quigley et al., 2002). Additionally, in adults, conscious emotion reappraisal can result in matching physiological changes (Gross, 1998). Taken together developmentally, individuals who perform well may learn to appraise less threat in future situations and this might increasingly promote more challenge physiological reactivity, resulting in matching stress responses. However, a third alternative is that these CP and TG groups of adolescents maintain this mismatched pattern of stress appraisal and reactivity through adulthood, accounting for a subset of adults that have less aligned stress responses. Future, longitudinal studies should follow adolescents into adulthood in order to determine which of these three outcome is more likely.

\section{Conclusion}

This study demonstrates that the relationships among stress appraisals, physiological reactivity, and performance differ among adolescents as compared to what has previously been observed in adults, highlighting adolescents' difficulties in accurately predicting and interpreting their responses to stress. Instead of finding support for the BPS stress model in adolescence, our findings imply that for adolescents it might be easier to use behavioral cues, such as one's performance ability, to appraise stressful situations than it is to interpret their physiological responses. This lack of awareness in adolescents might lead good performers to continuously place themselves in stressful situations, and even if they do not perceive such events as stressful, their body could still undergo the wear and tear of threat physiological reactivity. Future studies on stress responses in adolescence should seek to identify the cognitive, physiological, and behavioral factors that predict how stress responses in adolescence carry over into adulthood. Further investigation of the complicated nature of stress processes will elucidate the sources of misinterpretations of stress responses, and help individuals better understand and predict their stress responses in the most adaptive way. As more research examines the relationships between these stress variables, we can develop greater insights into answering the larger question of what factors drive the increases in stress that occur during adolescence. 


\section{References}

Allen JB, Howe BL. Player ability, coach feedback, and female adolescent athletes' perceived competence and satisfaction. J Sport Exerc Psychol. 1998; 20:280-99.

Blascovich J, Tomaka J. The biopsychosocial model of arousal regulation. Adv Exp Soc Psychol. 1996; 28:1-51.

Blascovich J, Mendes WB, Hunter SB, Salomon K. Social "facilitation" as challenge and threat. J Pers Soc Psychol. 1999; 77:68-77. [PubMed: 10434409]

Blascovich, J.; Mendes, WB. Challenge and threat appraisals: the role of affective cues. In: Forgas, J., editor. Feeling and thinking: the role of affect in social cognition. Cambridge, England: Cambridge University Press; 2000. p. 59-82.

Blascovich J, Mendes WB, Hunter SB, Lickel B, Kowai-Bel N. Perceiver threat in social interactions with stigmatized others. J Pers Soc Psychol. 2001; 80:253-67. [PubMed: 11220444]

Blascovich J, Seery MD, Mugridge CA, Norris RK, Weisbuch M. Predicting athletic performance from cardiovascular indexes of challenge and threat. J Exp Soc Psychol. 2004; 40:683-88.

Blascovich, J.; Vanman, E.; Mendes, WB.; Dickerson, S. Social psychophysiology for social and personality psychology. Los Angeles, CA: Sage; 2011.

Buske-Kirschbaum A, Jobst S, Wustmans A, Kirschbaum C, Rauh W, Hellhammer D. Attenuated free cortisol response to psychosocial stress in children with atopic dermatitis. Psychosom Med. 1997; 59:419-26. [PubMed: 9251162]

Davey CG, Yücel M, Allen NB. The emergence of depression in adolescence: development of the prefrontal cortex and the representation of reward. Neurosci Biobehav Rev. 2008; 32:1-19. [PubMed: 17570526]

Denscombe M. Social conditions for stress: young people's experience of doing GCSEs. Br Educ Res J. 2000; 26:359-74.

Dickerson SS, Kemeny ME. Acute stressors and cortisol responses: a theoretical integration and synthesis of laboratory research. Psychol Bull. 2004; 130:355-91. [PubMed: 15122924]

Drach-Zahavy A, Erez M. Challenge versus threat effects on the goal-performance relationship. Organ Behav Hum Decis Process. 2002; 88:667-82.

Folkman S, Lazarus RS, Gruen RJ, DeLongis A. Appraisal, coping, health status, and psychological symptoms. J Pers Soc Psychol. 1986; 50:571-79. [PubMed: 3701593]

Fydrich T, Chambless DL, Perry KJ, Buergener F, Beazley MB. Behavioral assessment of social performance: a rating system for social phobia. Behav Res Ther. 1998; 36:995-1010. [PubMed: 9714949]

Gogtay N, Giedd JN, Lusk L, Hayashi KM, Greenstein D, Vaituzis AC, Nugent TF, et al. Dynamic mapping of human cortical development during childhood through early adulthood. Proc Natl Acad Sci U S A. 2004; 101:8174-9. [PubMed: 15148381]

Gray HM, Mendes WB, Denny-Brown C. An in-group advantage in detecting intergroup anxiety. Psychol Sci. 2008; 19:1233-7. [PubMed: 19121129]

Gross JJ. Antecedent- and response-focused emotion regulation: divergent consequences for experience, expression, and physiology. J Pers Soc Psychol. 1998; 74:224-37. [PubMed: 9457784]

Grour MW, Thomas SP, Shoffner D. Adolescent stress and coping: a longitudinal study. Res Nurs Health. 1992; 15:209-17. [PubMed: 1509113]

Gunnar MR, Wewerka S, Frenn K, Long JD, Griggs C. Developmental changes in hypothalamuspituitary-adrenal activity over the transition to adolescence: normative changes and associations with puberty. Dev Psychopathol. 2009; 21:69-85. [PubMed: 19144223]

Hewitt MP. Self-evaluation accuracy among high school and middle school instrumentalists. J Res Music Educ. 2005; 53:148-61.

Hodgins HS, Weibust K, Weinstein N, Shiffman S, Miller A, Coombs G, Adair KC. The cost of selfprotection: threat response and performance as a function of autonomy and control motivation. Pers Soc Psychol Bull. 2010; 36:1101-14. [PubMed: 20693387]

Howard GS, Dailey PR. Response-shift bias: a source of contamination of self-report measures. J Appl Psychol. 1979; 64:144-50. 
Jamieson JP, Mendes WB, Blackstock E, Schmader T. Turning the knots in your stomach into bows: reappraising arousal improves performance on the GRE. J Exp Soc Psychol. 2010; 46:208-12. [PubMed: 20161454]

Jamieson JP, Nock MK, Mendes WB. Mind over matter: reappraising arousal improves cardiovascular and cognitive responses to stress. J Exp Psychol Gen. 2012; 141:417-22. [PubMed: 21942377]

Jones M, Meijen C, McCarthy PJ, Sheffield D. A theory of challenge and threat states in athletes. Int Rev Sport Exerc Psychol. 2009; 2:161-80.

Kassam KS, Koslov K, Mendes WB. Decisions under distress: stress profiles influence anchoring and adjustment. Psychol Sci. 2009; 20:1394-99. [PubMed: 19843261]

Kelsey RM, Blascovich J, Leitten CL, Schneider TR, Tomaka J, Wiens S. Cardiovascular reactivity and adaptation to recurrent psychological stress: moderating effects of evaluative observation. Psychophysiol. 2000; 37:748-56.

Kerr S, Johnson VK, Gans SE, Krumrine J. Predicting adjustment during the transition to college: alexithymia, perceived stress and psychological symptoms. J Coll Stud Dev. 2004; 45:593-607.

Kirschbaum C, Pirke KM, Hellhammer D. The 'trier social stress test' - a tool for investigating psychobiological stress responses in a laboratory setting. Neuropsychobiology. 1993; 28:76-81. [PubMed: 8255414]

Kopp CB. Regulation of distress and negative emotions: a developmental view. Dev Psychol. 1989; 25:343-54.

Kudielka, BM.; Hellhammer, DH.; Kirschbaum, C. Ten years of research with the trier social stress test - revisited. In: Harmon-Jones, E.; Winkielman, P., editors. Social neuroscience: integrating biological and psychological explanations of social behavior. New York: The Guilford Press; 2007. p. 56-83.

Larson R, Ham M. Stress and" storm and stress" in early adolescence: the relationship of negative events with dysphoric affect. Dev Psychol. 1993; 29:130-40.

McCormick C, Mathews I. HPA function in adolescence: role of sex hormones in its regulation and the enduring consequences of exposure to stressors. Pharmacol Biochem Behav. 2007; 86:220-33. [PubMed: 16901532]

Mendes WB, Blascovich J, Major B, Seery MD. Challenge and threat responses during downward and upward social comparisons. Eur J Soc Psychol. 2001; 31:477-97.

Mendes WB, Reis HT, Seery MD, Blascovich J. Cardiovascular correlates of emotional expression and suppression: do content and gender context matter? J Pers Soc Psychol. 2003; 84:771-92. [PubMed: 12703648]

Mendes WB, Gray HM, Mendoza-Denton R, Major B, Epel E. Why egalitarianism might be good for your health: physiological thriving during intergroup interactions. Psychol Sci. 2007; 18:991-8. [PubMed: 17958714]

Mendes WB, Major B, McCoy S, Blascovich J. How attributional ambiguity shapes physiological and emotional responses to social rejection and acceptance. J Pers Soc Psychol. 2008; 94:278-91. [PubMed: 18211177]

Monroe SM. Modern approaches to conceptualizing and measuring human life stress. Ann Rev Clin Psychol. 2008; 4:33-52. [PubMed: 17716038]

Moore LJ, Vine SJ, Wilson MR. The effect of challenge and threat states on performance: an examination of potential mechanisms. Psychobiol. 2012; 49:1417-25.

Moos RH, Holahan CJ. Dispositional and contextual perspectives on coping: toward an integrative framework. J Clin Psychol. 2003; 59:1387-403. [PubMed: 14618606]

Nicholls JG. Achievement motivation: conceptions of ability, subjective experience, task choice, and performance. Psychol Rev. 1984; 91:328-46.

Ochsner KN, Knierim K, Ludlow DH, Hanelin J, Ramachandran T, Glover G, Mackey SC. Reflecting upon feelings: an fMRI study of neural systems supporting the attribution of emotion to self and other. J Cogn Neurosci. 2004; 16:1746-72. [PubMed: 15701226]

Ordaz S, Luna B. Sex differences in physiological reactivity to acute psychosocial stress in adolescence. Psychoneuroendocrinology. 2012; 37:1135-57. [PubMed: 22281210]

Pennebaker, JW. The psychology of physical symptoms. New York: Springer-Verlag; 1982. 
Phelan P, Yu HC, Davidson AL. Navigating the psychosocial pressures of adolescence: the voices and experiences of high school youth. Am Educ Res J. 1994; 31:415-47.

Philippot P, Segal Z. Mindfulness based psychological interventions: developing emotional awareness for better being. J Conscious Stud. 2009; 16:285-306.

Quested E, Bosch JA, Burns VE, Cumming J, Ntoumanis N, Duda JL. Basic psychological need satisfaction, stress-related appraisals, and dancer's cortisol and anxiety responses. J Sport Exerc Psychol. 2011; 33:828-46. [PubMed: 22262707]

Quigley KS, Barrett LF, Weinstein S. Cardiovascular patterns associated with threat and challenge appraisals: a within-subjects analysis. Psychophysiol. 2002; 39:292-30.

Romeo RD, McEwen BS. Stress and the adolescent brain. Ann N Y Acad Sci. 2006; 1094:202-14. [PubMed: 17347352]

Salovey, P.; Mayer, JD.; Goldman, SL.; Turvey, C.; Palfai, TP. Emotional attention, clarity, and repair: exploring emotional intelligence using the Trait Meta-Mood Scale. In: Pennebaker, JW., editor. Emotion, disclosure, and health. Washington, DC: American Psychological Association; 1995. p. 125-54.

Sanders AF. Towards a model of stress and human performance. Acta Psychol. 1983; 53:61-97.

Schmader T, Johns M, Forbes C. An integrated process model of stereotype threat on performance. Psychol Rev. 2008; 115:336-56. [PubMed: 18426293]

Schneider TR. Evaluations of stressful transactions: what's in an appraisal? Stress Health. 2008; 24:151-8.

Schneiderman N, Ironson G, Siegel SD. Stress and health: psychological, behavioral, and biological determinants. Ann Rev Clin Psychol. 2005; 1:607-28. [PubMed: 17716101]

Seery MD. Challenge or threat? Cardiovascular indexes of resilience and vulnerability to potential stress in humans. Neurosci Biobehav Rev. 2011; 35:1603-10. [PubMed: 21396399]

Seiffge-Krenke I, Weidemann S, Fentner S, Aegenheister N, Poeblau M. Coping with school-related stress and family stress in healthy and clinically referred adolescents. Eur Psychol. 2001; 6:12332.

Sherwood A, Kelsey R, Lovallo WR, van Doornen LJP. Methodological guidelines for impedance cardiography. Psychophysiol. 1990; 27:1-23.

Sowell ER, Trauner DA, Gamst A, Jernigan TL. Development of cortical and subcortical brain structures in childhood and adolescence: a structural MRI study. Dev Med Child Neurol. 2002; 44:4-16. [PubMed: 11811649]

Spear LP. Heightened stress responsivity and emotional reactivity during pubertal maturation: implications for psychopathology. Dev Psychopathol. 2009; 21:87-97. [PubMed: 19144224]

Steinberg L. Cognitive and affective development in adolescence. Trends Cogn Sci. 2005; 9:69-74. [PubMed: 15668099]

Steinberg L, Morris AS. Adolescent development. Ann Rev Psychol. 2001; 52:83-110. [PubMed: 11148300]

Stroud LR, Foster E, Papandonatos GD, Handwerger K, Granger DA, Kivlighan KT, Niaura R. Stress response and the adolescent transition: performance versus peer rejection stressors. Dev Psychopathol. 2009; 21:47-68. [PubMed: 19144222]

Sze JA, Gyurak A, Yuan JW, Levenson RW. Coherence between emotional experience and physiology: does body awareness training have an impact? Emotion. 2010; 10:803-14. [PubMed: 21058842]

Tomaka J, Blascovich J, Kelsey RM, Leitten CL. Subjective, physiological, and behavioral effects of threat and challenge appraisal. J Pers Soc Psychol. 1993; 65:248-60.

Willard G, Gramzow RH. Beyond oversights, lies, and pies in the sky: exaggeration as goal projection. Pers Soc Psychol Bull. 2009; 35:477-92. [PubMed: 19171773]

Williams SE, Cumming J, Balanos GM. The use of imagery to manipulate challenge and threat appraisals in athletes. J Sport Exerc Psychol. 2010; 32:339-58. [PubMed: 20587822]

Wirtz PH, von Kanel R, Mohiyeddini C, Emini L, Ruedisueli K, Groessbauer S, Ehlert U. Low social support and poor emotional regulation are associated with increased stress hormone reactivity to mental stress in systemic hypertension. J Clin Endocrinol Metabol. 2006; 91:3857-65. 
Zuddas A. A crucial role for basic emotion awareness in the development of emotion regulation? Eur Child Adolesc Psychiatr. 2012; 21:297-9. 


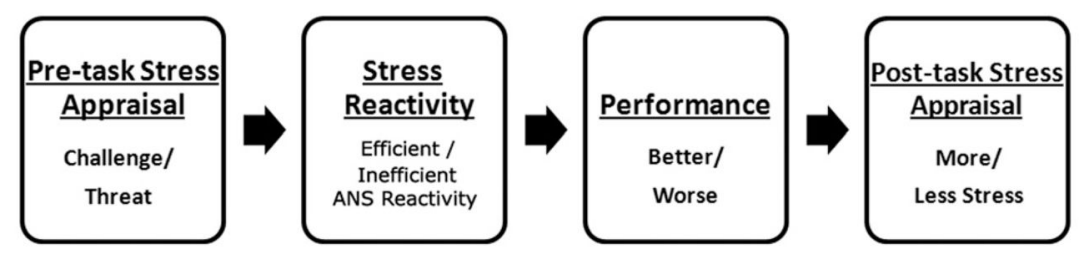

Figure 1.

The linear relationships between variables in the biopsychosocial model of challenge and threat. The variables ultimate lead to post-task stress appraisal, here a result of the preceding stress appraisal, physiological reactivity, and performance. 


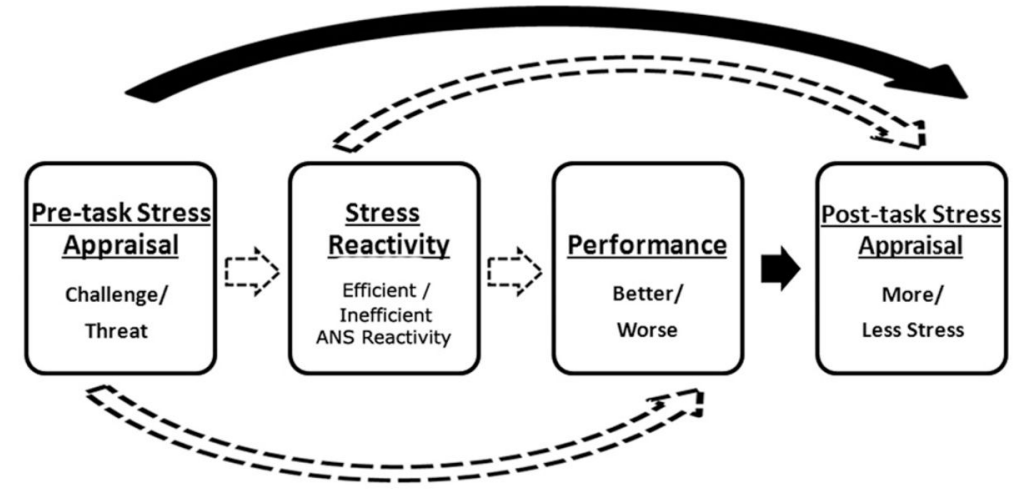

Figure 2.

The statistically significant relationships found between variables in the biopsychosocial model of challenge and threat. Bold arrows indicate significant relationships found in our sample of adolescents. Dotted arrows indicate relationships found by past research on the BPS model in older populations that were not replicated in this study. 


\section{Table 1}

Means and standard deviations of stress appraisal, stress reactivity, and performance variables.

\begin{tabular}{lrr}
\hline & Mean & SD \\
\hline Pre-task stress appraisal & 10.57 & 4.19 \\
Baseline heart rate & 73.24 & 11.12 \\
Baseline cardiac output & 6.06 & 2.11 \\
Baseline TPR & 1176.31 & 528.88 \\
Speech heart rate & 100.45 & 20.76 \\
Speech cardiac output & 6.83 & 2.40 \\
Speech TPR & 1321.60 & 659.94 \\
Stress reactivity $\Delta$ CO & 0.77 & 1.15 \\
Stress reactivity $\Delta$ TPR & 139.30 & 290.68 \\
Performance & 36.37 & 9.19 \\
Post-task stress appraisal & 12.16 & 4.12 \\
\hline
\end{tabular}




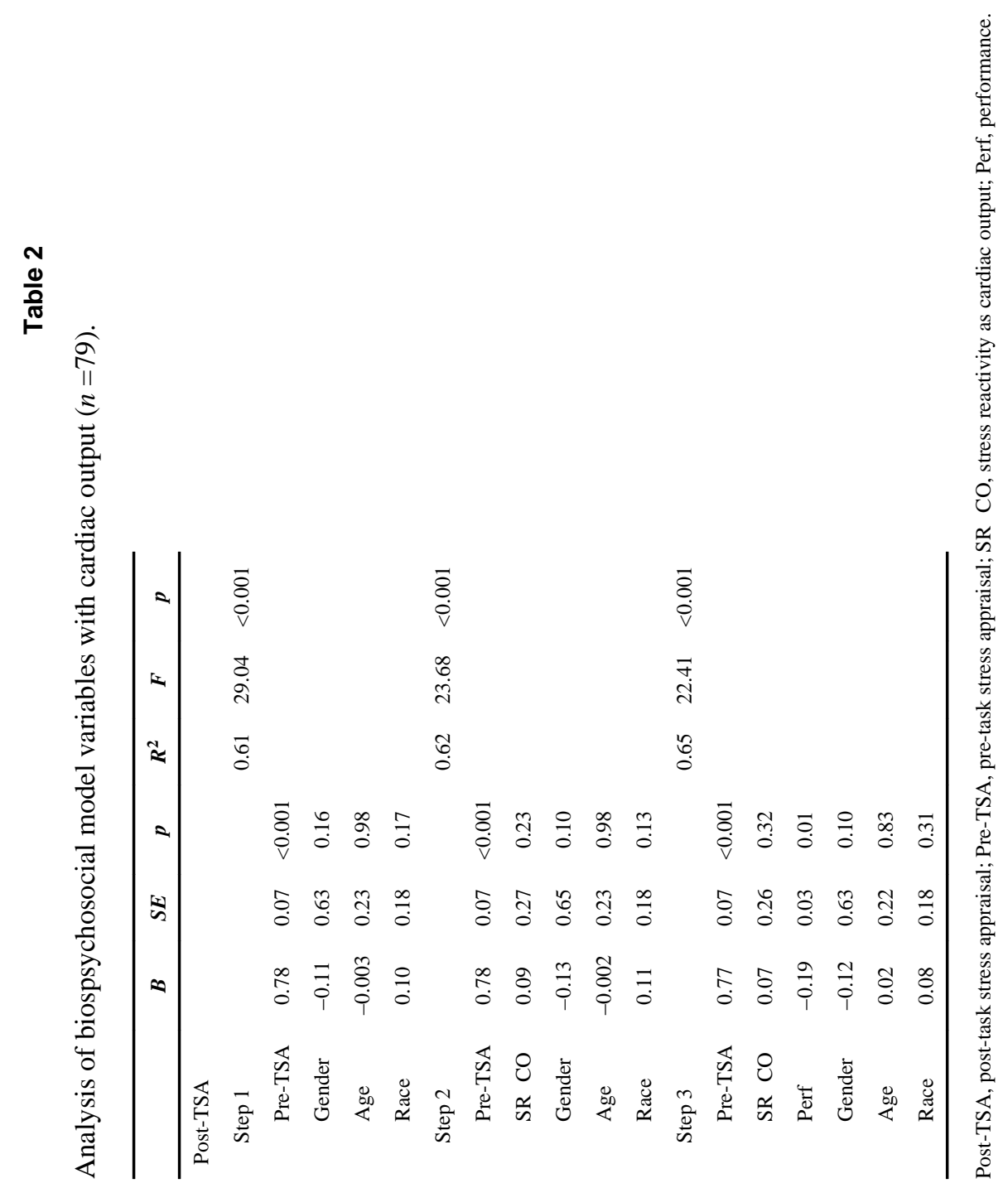




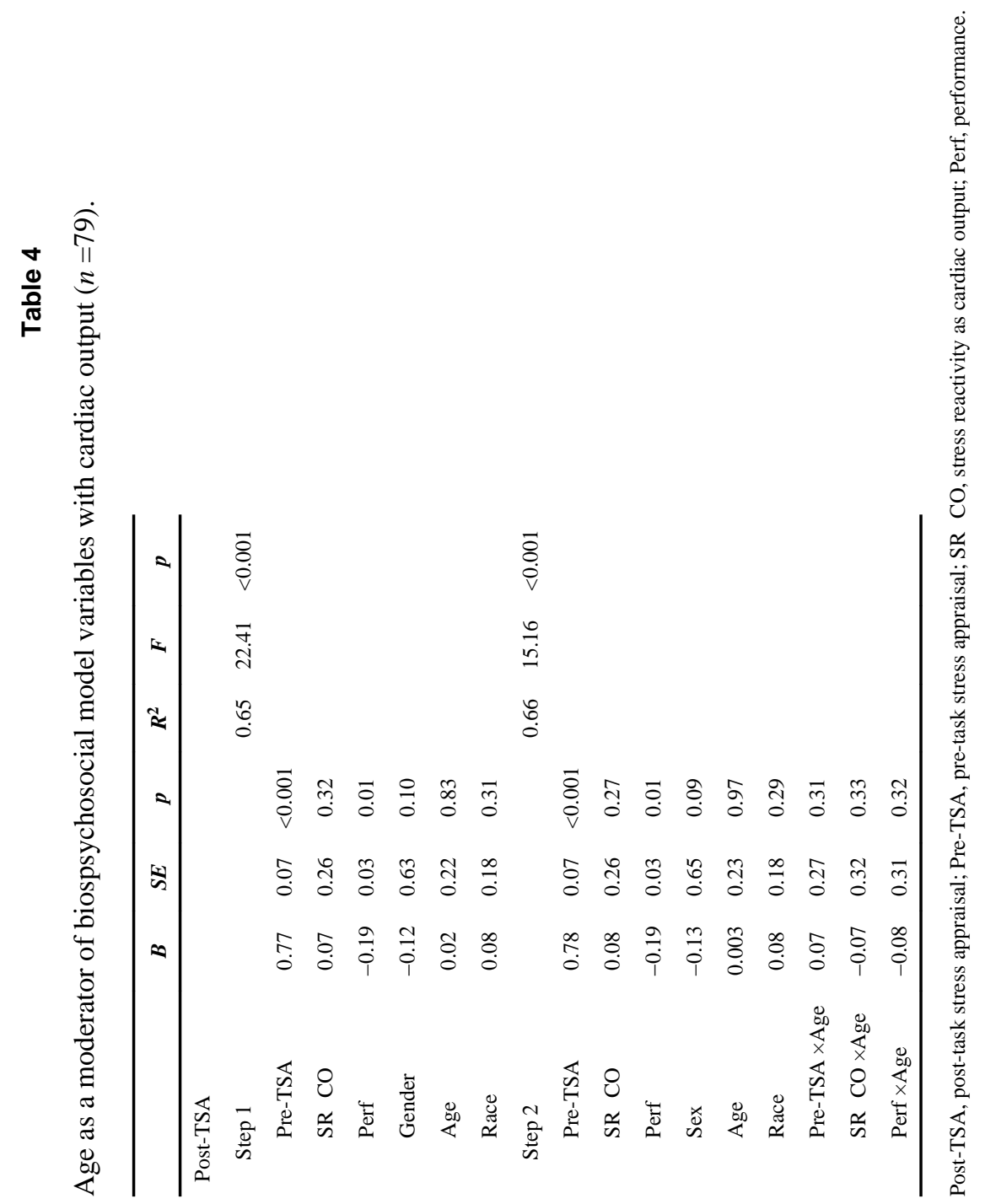




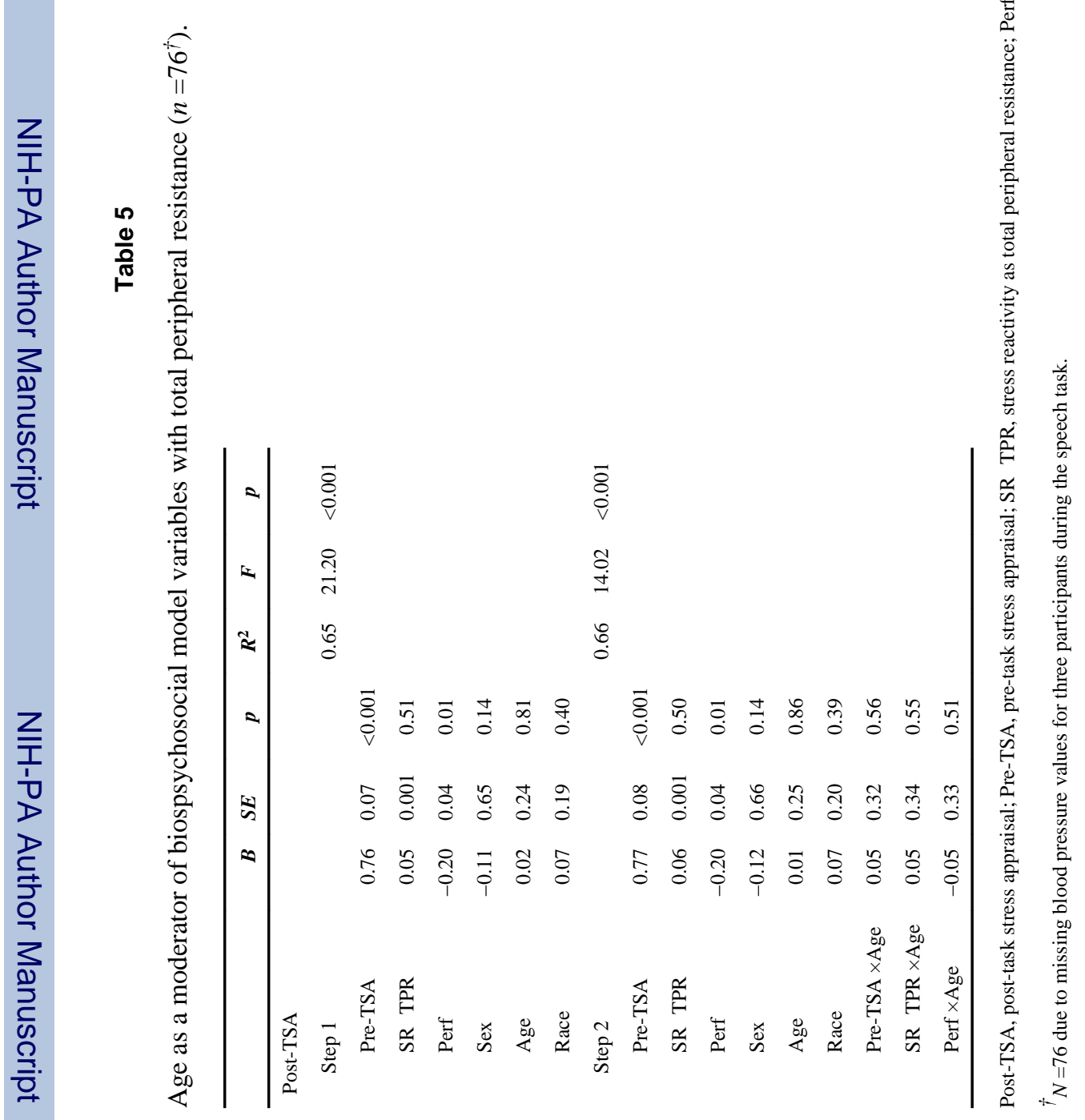

